\title{
The use of enzymes for construction of DNA-based objects and assemblies $\dagger$
}

\author{
Sascha Keller and Andreas Marx* \\ Received 10th February 2011 \\ DOI: $10.1039 / \mathrm{c} 1 \mathrm{cs} 15040 \mathrm{e}$
}

DNA has found wide applications in DNA-based nanotechnology due to its simplicity and predictability of its secondary structure. Selecting DNA for the nanoconstruction of objects and assemblies bears the inherent potential for manipulations and control by DNA modifying enzymes. In this tutorial review, we present an overview of the enzyme-catalysed construction of DNA-based objects and assemblies. It is illustrated how a diversity of enzyme-based biochemical reactions are transferred in nanotechnological applications.

\section{Introduction}

Besides the natural purpose of DNA as an information encoding biopolymer, DNA-based nanotechnology uses DNA as a scaffold for the generation of nanometre- to micrometre-scale structures. DNA has found wide applications in DNA-based nanotechnology due to its simplicity and predictability of its secondary structure. ${ }^{1}$ Thereby, DNA nanoarchitectures are mainly based on intelligent sequence design leading to defined hybridised structures out of short chemically synthesised oligodeoxynucleotides (ODNs). Based on this principle a variety of two- and three-dimensional nano-objects was reported. ${ }^{2,3}$ To enlarge the dimension of DNA-based structures periodical

Department of Chemistry and Konstanz Research School Chemical Biology, University of Konstanz, 78457 Konstanz, Germany.

E-mail: Andreas.Marx@uni-konstanz.de; Fax: + 49753188 5140;

Tel: +497531885139

$\dagger$ Part of a themed issue on the advances in DNA-based nanotechnology. repeats of nano-objects were successfully employed to reach the microscopic scale. ${ }^{4,5}$ Chemical self-replication in solution ${ }^{6}$ and on surfaces ${ }^{7}$ was used to amplify nano-objects. In future, this concept might circumvent the scale-up processes that hitherto limit broad applications.

One prospective advantage of DNA for nanoconstruction is the inherent potential for generation and manipulation by DNA modifying enzymes. This topic is only at the beginning of being fully explored. The application of enzymes in nanotechnology, with its myriad of tools available for manipulation of DNA in a sequence specific manner, holds great potential for using DNA as a generic material. Enzymes are highly selective and processive nanofactories in biological processes. Over the last few decades, many DNA-modifying enzymes have been characterised and a huge variety of possible substrates is commercially available. Up to now, the development of enzyme catalysed construction of DNA-based nanostructures has been focused on implementing well-known commercially

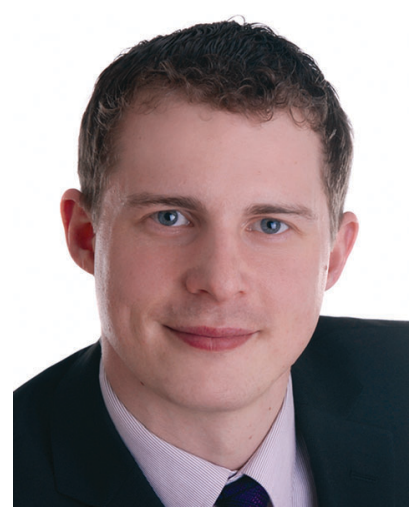

Sascha Keller
Born in 1981 in Pfullendorf (Germany), Sascha Keller studied chemistry at the University of Konstanz (Germany) and finished his study in Konstanz with his diploma thesis in 2006 in the group of Prof. A. Marx. Then he has been working as a graduate student on the topic of enzyme mediated network growth using chemically modified oligonucleotides. Currently, he moved to industry where he is working as a study director for residue analysis at Eurofins Agroscience Services.

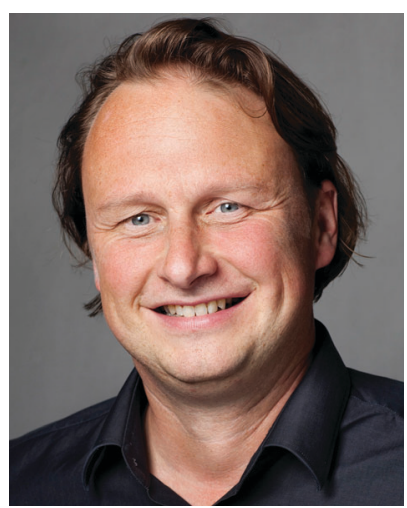

Andreas Marx
Andreas Marx studied chemistry in Freiburg (Germany), Sussex (UK), and Bochum (Germany). In 1997 he earned his DPhil at Basel University (Switzerland). After a postdoctoral time at Nagoya University (Japan) he began his independent career at the University of Bonn (Germany) 1999. In 2004 he moved to the University of Konstanz (Germany) as a full-professor in chemistry and currently holds the Chair of Organic Chemistry and Cellular Chemistry. His research focuses on the chemical biology of DNA replication processes. 
available enzymes that are briefly introduced in this chapter. For nanotechnology the enzymatic synthesis of long DNA strands offers the chance to expand the scale dimensions of the bottom-up approaches and opens the door to extended assemblies up to millimetre scale. Thereby, the enzyme family of ligases engaged an important function. Ligases covalently connect the $5^{\prime}$-phosphate group of one strand and the $3^{\prime}$-hydroxyl group of another strand, thus forming a phosphodiester bond allowing to connect multiple DNA strands. As an antagonist, restriction enzymes catalyse sequence specific cleavage of phosphodiester bonds, thereby releasing short single stranded DNA sequences. Another useful enzyme is the terminal deoxynucleotidyl transferase (TdT). TdT is able to extend template independently a DNA strand from its $3^{\prime}$-hydroxy terminus, thus generating single stranded DNA (ssDNA). Furthermore, DNA polymerases represent an important class of enzymes. These are mainly used in the polymerase chain reaction (PCR), presenting a tool for the exponential amplification of DNA material in a short time scale. Considering scaling up processes, DNA polymerases are a class of enzymes that could play an important role in further developments. Beyond that, several DNA polymerases can even proceed on circularized templates producing a periodical sequence in the rolling circle amplification (RCA) and therefore are a candidate for scaling up processes or replicating in enzyme-based nanotechnology.

In this tutorial review we present examples in the field of DNA-based nanotechnology in which DNA-modifying enzymes were employed.

\section{Enzyme-catalysed manipulation of DNA on self-assembled monolayers (SAM)}

The ability to carry out biochemical reactions catalysed by enzymes with nanoscale precision on surfaces is an important goal in the development of bottom-up nano-manufacturing. Thereby, Chilkoti and co-workers described a proof of principle approach of an enzyme-catalysed reaction on an ODN modified Au-surface (Fig. 1.1). ${ }^{7}$ Their approach is based on self-assembled monolayers (SAM) presenting terminal ODNs. The catalytic enzyme is adsorbed on an atomic force microscope (AFM) tip.

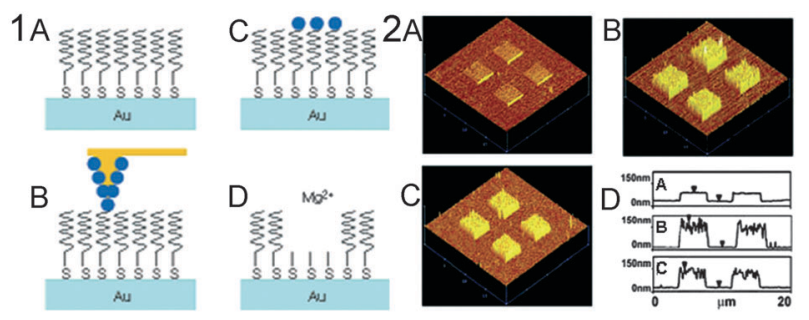

Fig. 1 Two examples for enzymatic manipulation on SAM: (1) ODN terminated SAM on Au get selectively digested (A), AFM-tip inked with DNAseI (B), adsorbed enzyme on SAM (C), local digest of DNA induced by $\mathrm{Mg}^{2+}$ ions in solution (D). ${ }^{7}$ (Reprinted with permission from ref. 7.) (2) AFM images of gold arrays with $5^{\prime}-\mathrm{SH}-\left(\mathrm{CH}_{2}\right)_{6}-\mathrm{T}_{25}$ DNA-SAM: after treated with heat-inactivated TdT (A), active TdT (B), and active TdT followed by exonuclease I digestion (C). The line profiles of these figures are shown in $(D)^{8}$ (reproduced with permission from ref. 8).
Taking benefit of the well-evolved dip-pen nanolithography (DPN), ${ }^{9}$ it was possible to deposit the nuclease DNAseI with nanometre precision on the self-assembled monolayers. Digestion was initiated in the next step by coating the surface with a magnesium-ion containing buffer. This led to nanotrenches observed by AFM. In this case, DNAseI was able to modify a DNA assembly on the surface with nanometre precision.

Further investigations by the same group employ TdT for extension of DNA on surfaces (Fig. 1.2). ${ }^{8}$ Instead of digesting an existing monolayer of DNA, they extend template independently a self-assembled monolayer consisting of ODNs. TdT is able to extend ODN strands that are nanopatterned on the surface. Thereby, the enzyme is freely diffusing in solution which also differs from the above mentioned DPN method. Further, the extended DNA could be digested afterwards by exonuclease I (Fig. 1.2C). Next, the TdT properties on selfassembled monolayers were studied in more detail. ${ }^{10}$ Thereby, a dependence on the extension process by mainly two factors was observed. First, the surface-initiated enzymatic polymerization process showed clear regulation depending on the density and thickness of self-assembled ODN monolayers. A thick and dense monolayer results in less extension efficiency of the TdT related to sterical problems appearing on the surface. On the other hand, the choice of used nucleotides showed significant influence on the extension behaviour as well. The authors speculate about extending the approach by introducing modified nucleotides acting as affinity tags. Doing so, functional molecular scaffolds might be generated to dock nanoparticles and biomolecules on a surface in order to fabricate hybrid structures at the meso-microscale and to provide new supramolecular architectures for biosensors and clinical diagnostics. Another approach for enzyme mediated manipulations of ODN immobilized on surfaces was reported by Castronovo and co-workers. ${ }^{11}$ They demonstrated that sequence specific digestion catalysed by DpnII was possible but dependent on the density of immobilised double-stranded DNA (dsDNA). It was observed that efficient digestion only appeared if the distance between two ODNs was twofold the diameter of the used restriction enzyme. In principle, the approach illustrated that restriction enzymes can be used with modified parameters for manipulations of DNA assemblies on self-assembled monolayers. Recently, Kaufmann and co-workers reported the consecutive use of enzymes for manipulations of self-assembled monolayers. ${ }^{12}$ They realised their concept of generating a patterned surface bearing different, spatially separated DNA sequences. These sequences were processed by using a restriction enzyme $($ Bam $\mathrm{HI})$ and a DNA polymerase I (Klenow fragment of $E$. coli DNA polymerase I). Thereby, nanopatterned ODNs with different sequences are selectively digested first. Resulting DNA layers allowed sequencespecific extension by addition of modified nucleotides.

The discussion of enzymatic manipulations concerning nanopatterned DNA on surfaces illustrates that enzymes have a high impact on sequence specific manipulations by restriction, digestion and template dependent and independent sequence extensions. Furthermore, a DNA polymerase was reported which incorporates modified nucleotides in a DNA strand surrounded by dense surface-bound long DNA strands. ${ }^{12}$ 
The use of enzymes and their precise action demonstrates their potential for further applications in surface structuring.

\section{Enzymatic impact on DNA modified nanoparticles}

The fact that optical properties of Au-nanoparticles (AuNP) are governed by the size of AuNP aggregates induced a variety of approaches of DNA-controlled assembly strategies. The pioneering work by Mirkin and his co-workers on DNA-mediated assemblies of AuNP has opened the door to a variety of potential applications in biological sensing, medical diagnostics and drug delivery. ${ }^{13}$ Along these lines, researchers used enzymes as DNA specific and regulating tools for AuNP aggregation.

Kanaras and co-workers adapted enzyme catalysed manipulations of DNA for the generation of nanoscale building blocks based on AuNPs. Thereby, they used the stabilising effect of surface-bound ODN on AuNP in solution. DNA bound to AuNP can be described as a protecting moiety for spontaneous aggregation of nanoparticles (Fig. 2). ${ }^{14}$ They extended the existing approach of assembling single stranded DNA-capped AuNPs by sequence directed hybridisation to a programmed assembly approach which could be regulated by restriction enzymes. Doing so, AuNPs were coated with a ligand shell of thio-modified single stranded DNA (ssDNA), allowing a complementary DNA strand to hybridise with the ssDNA on the surface and to form a stable duplex.

This resulted in modified AuNPs that were not able to assemble with another AuNP. Further, they designed the DNA strands in a way that a recognition site for EcoRI was included (Fig. 2A). Doing so, the used restriction enzyme EcoRI was able to cleave the double strands sequence specifically and release cohesive ends capable for new hybridisation events resulting in AuNP aggregates (Fig. 2B). In principle, this concept can be transferred to a myriad of other restriction enzymes thereby controlling assembly processes. The characterization of AuNP assemblies was monitored by a visible colour change induced by aggregation. As a next step, the hybridised AuNP assemblies were further stabilised with T4 DNA ligase (Fig. 2C). ${ }^{14}$ Thus, T4 DNA ligase catalysed the formation of phosphodiester bonds and increased the thermal stability of the hybridised

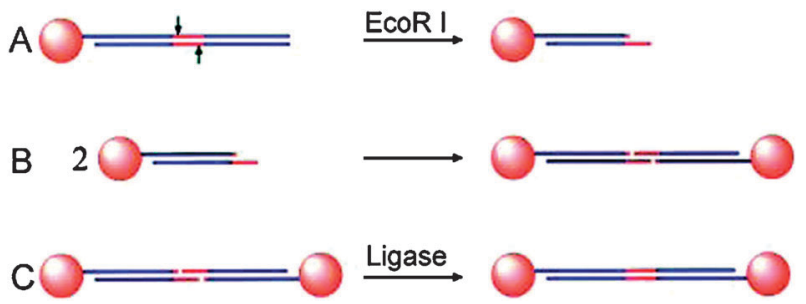

Fig. 2 Schematic description of the method. (A) Gold nanoparticles derivatized with double-stranded DNA are treated with restriction enzyme EcoRI, which cleaves the DNA to yield cohesive ends. The red color represents the recognition site of the enzyme, and the arrows indicate the sites of cleavage on each strand. In reality, each $15 \mathrm{~nm}$ particle has around 100 DNA ligands; (B) two cohesive ends hybridise leading to a weak association of particles; (C) the DNA backbones are covalently joined at the hybridised site by DNA ligase to yield a double-stranded link between particles ${ }^{14}$ (reproduced with permission from ref. 14). assemblies. Further studies indicated that this process is reversible and the ligated DNA-AuNP aggregates can be subsequently cleaved by treatment with a restriction enzyme. ${ }^{15}$ The system was put forward to a dynamic system in which hybridisation of ODN-modified AuNP was followed by a ligation step and a restriction digest illustrating that the dynamic control of AuNP assembly processes can be controlled by enzymatic reactions. ${ }^{16}$ This nanostructure manipulation with enzymes provides an additional control of selective cleavage and ligation over nanostructure assembly. Up to date, a plethora of restriction enzymes has been discovered and hundreds are commercially available. This allows for a large number of selective preparations of nanostructure building blocks in future.

The number of enzymes adaptable to DNA-modified AuNP was increased by the Klenow fragment of $E$. coli DNA polymerase I. This enzyme was identified to extend an ODN bound to AuNP with the help of a hybridised template strand (Fig. 3A). ${ }^{17}$ It was found that the enzyme elongation efficiency was strongly dependent on the density of ODN bound to the AuNP and the linker length that connects the ODN with the Au surface. These sterical problems also influenced the efficient primer-template annealing which resulted in decreased extension efficiency.

One example for amplifying AuNP species connected with ssDNA rather than dsDNA was reported by Claridge and co-workers. ${ }^{18}$ The researchers illustrated an approach called ligase chain reaction (LCR). In LCR, two strands of DNA were first hybridised to a DNA template to form a nicked double strand region. The reaction mixture was afterwards incubated to allow Thermus aquaticus DNA ligase to form a covalent bond at the nick site. Afterwards, the reaction
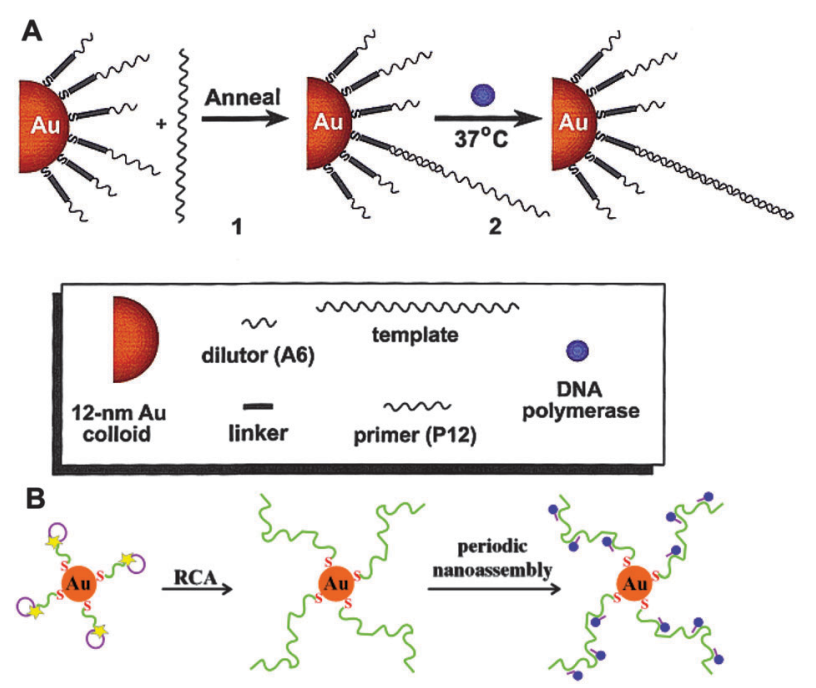

Fig. 3 (A) In step 1, the primers in the DNA-AuNP conjugates are annealed to the template strand followed by extension in step 2 accomplished by the addition of Klenow (the large fragment of DNA polymerase I) ${ }^{17}$ (reproduced with permission from ref. 17). (B) Schematic illustration of RCA on AuNPs. DNA-AuNP conjugates produced by RCA as a scaffold templating the binding of the formation of $3 \mathrm{D}$ nanostructures (blue dots) ${ }^{19}$ (reproduced with permission from ref. 19). 
mixture was heated to melt the template from the ligated dimers. Thereby, AuNP dimers bridged by ssDNA linkers were formed and released cycle by cycle.

Zhao and co-workers adapted another idea to DNA modified AuNP. ${ }^{19}$ They disclosed the performance of rolling circle amplification (RCA) on AuNP (Fig. 3B). RCA is known to be a straightforward biochemical method that can generate long ssDNA with repeating sequence units. First, thio-modified DNA primers were attached to AuNP and a circular template was annealed to the primer. Thereby, DNA polymerase $\Phi 29$, known to be proficient in RCA, was used to generate long ssDNA strands which were visualized by atomic force microscopy (AFM). ${ }^{20}$ The resulting ssDNA scaffolds were further modified by nanospecies like AuNP bearing complementary ODN. The ODNs were designed to hybridize in an ordered manner on the repeating template sequence forming an ordered three-dimensional assembly. Besides AuNP based progress, another class of nanoparticles appeared to be a target for enzymatic manipulations. Amphiphilic organic nanoparticles have great potential for future applications. ${ }^{21}$ DNA block-copolymers (DBC) built out of a hydrophobic blockcopolymer and polar DNA part were synthesised. These DBCs exhibit a shell of DNA and a core with a hydrophobic polymer in aqueous environment. ${ }^{22}$ Such DBC micelles have been investigated for potential applications in antisense oligonucleotide or drug delivery ${ }^{23}$ and combinatorial tools in nanotechnology-based therapeutic approaches. ${ }^{24}$ Recently, based on DBCs a virus-like particle with the potential to act as a bioinspired container was reported. ${ }^{25}$ In this context, enzymes showed to be a powerful tool for the regulation of particle and micelle growth. DBCs were successfully treated with a TdT. TdT extends ssDNA template independently at the $3^{\prime}$-hydroxy terminus leading to a post-synthetic extension of block copolymer aggregates. ${ }^{26}$ Thereby, the methods offered a possible control over the growth of nanoparticles by employing TdT under mild and isothermal conditions. ${ }^{27}$ Further, it was possible to observe micelle growth by scanning force microscopy (SFM) in a time dependent fashion.

\section{Enzymatic generation of DNA-based hydrogels}

Besides the use of DNA for assemblies and hybrid materials, DNA itself may be regarded as a material. Natural DNA molecules are commonly either linear or circular. However, in DNA recombination events branched DNA topologies, so called Holliday junctions, are formed. They have inspired researchers in the field of DNA nanotechnology in the design of rigidified building blocks with predictable topology by removing the sequence symmetry of the Holliday junction around the branch point to make it immobile against branch migration. ${ }^{28}$ The branched DNA constructs, so called tiles, have been employed numerous times e.g. for the bottom-up construction of structured DNA-based lattices. ${ }^{29} \mathrm{Li}$ and co-workers combined this concept to construct Y-shaped structure (Fig. 4A) and studied their self-assembly towards dendrimer-like DNA (DL-DNA). ${ }^{30}$ The Y-structure consists of three partial complementary ssDNAs that hybridise by crossover in a programmable fashion to form a distinct structure. For a controlled assembly to a DL-DNA, sticky ends were
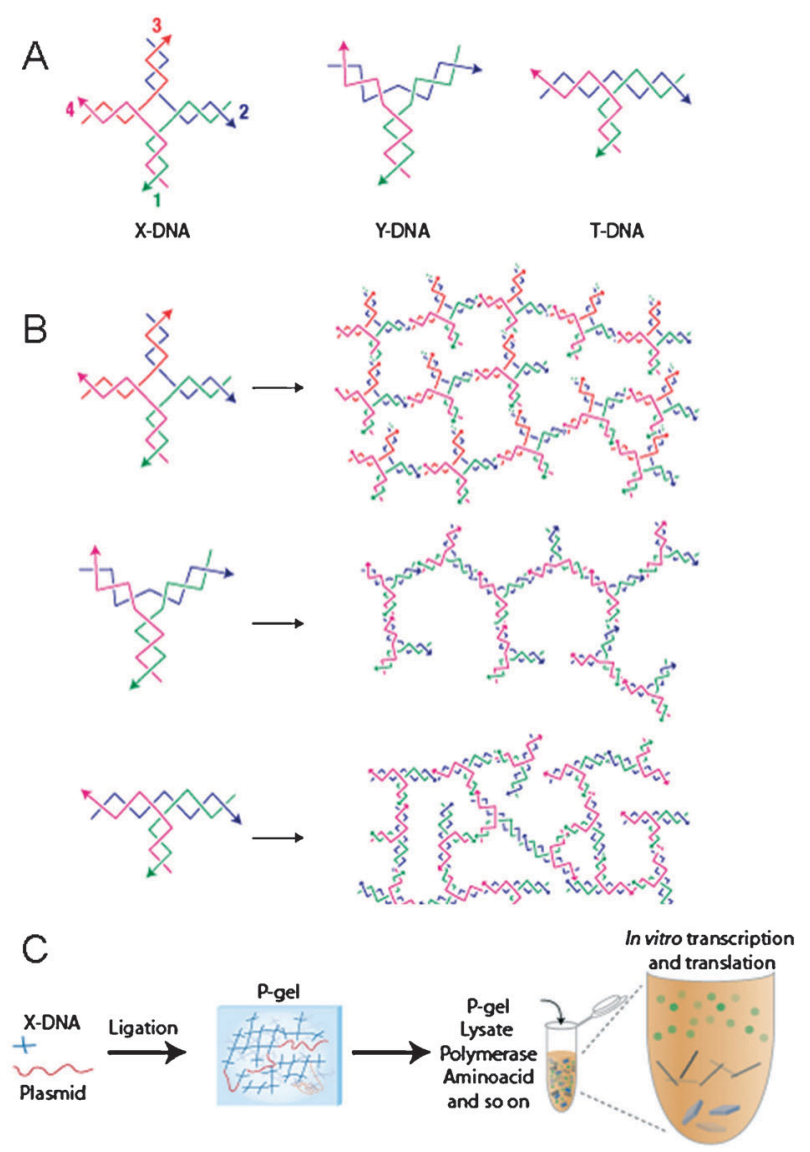

Fig. 4 (A) Used junctions, (B) crosslinking of Holliday-junctions via enzymatic ligation, ${ }^{31}$ (C) a schematic diagram of the gelation process through enzymatic crosslinking and cell-free expression with P-gel pads with a commercial transcription assay ${ }^{32}$ (reprinted by permission from ref. 31 and 32).

implemented in the design for specific binding to a complementary ssDNA. For example, a Y-shaped structure (compare Fig. 4A) was formed by hybridisation of three ssDNA strands into a three-way-junction bearing three sticky ends. As a consequence, the junction presented sticky ends for hybridisation. Thus, it was possible to assemble sequence-specifically another three Y-shaped DNA motifs resulting in a dendrimer-like structure. The authors called this step the first generation of assembly which was stabilised by enzymatic ligation. ${ }^{30}$ Following the aforementioned concept, it was possible to generate stable DL-DNA up to the fifth generation in a highly controlled fashion. This design and assembly strategy was transferred to construct other DNA building blocks that can be incorporated into even more complex nanostructures. In this context, the same research group extended the variety of branched DNA motifs with T- and X-DNA structures (Fig. 4A). ${ }^{31}$ These structures bear self-complementary sticky ends that resulted in the formation of complex networks (Fig. 4B). The network building blocks were covalently connected by enzyme-mediated ligation resulting in DNA-based hydrogels. Thereby, the intrinsic geometrical character of the DNA branching motifs significantly influenced the morphology of the DNA hydrogels. Further, interesting properties like biocompatibility, biodegradability and easy handling for fashioning in fabrication 
processes were observed. DNA scaffolds up to millimetre scale were possible to generate. The authors demonstrated several applications based on DNA-hydrogels like the encapsulation of mammalian cells. These cells can be recovered alive by a nuclease digestion after storage in the DNA-hydrogel for several days. In summary, the authors reported a new class of DNA-based material that might be exploited in a variety of applications including drug delivery, tissue engineering, 3D cell culture that might be useful in cell transplant therapy. ${ }^{31}$

Beside these studies, the same research group reported a further usage of DNA-based hydrogels. ${ }^{32}$ They successfully designed a cell-free protein-producing gel (P-gel). The concept is based on the generation of a hydrogel with X-shaped DNA (X-DNA) and long DNA strands that harbour the gene of a protein as building blocks (Fig. 4C). In this approach ligases were employed for the covalent connection of X-DNA structures and the DNA bearing the respective genes. Covalent connection resulted in the gel formation. In three studied examples the genes were successfully translated into proteins using a commercially available cell-free transcription and translation system. Interestingly, the amount of generated protein by the gel was higher than the amount generated by the corresponding translation of the plasmid in solution. This finding might have an impact on high-throughput protein engineering and protein production which could be realised in the near future.

\section{Enzyme-catalysed DNA network growth}

Besides the possible amplification of DNA nanojunctions via RCA, the polymerase chain reaction (PCR) holds the potential to be an alternative method. Recently, an approach was reported using Taq DNA polymerase to catalyse the generation of three-dimensional DNA networks via PCR (Fig. 5). ${ }^{33}$ The approach is based on the combination of covalently connected branched DNA (bDNA) Y-motifs that act as primer strands in PCR. The distances between the branched points were tuned using templates with different lengths ranging from oligonucleotides to entire genes. The approach to develop PCRamplifiable three-dimensional DNA networks is based on branched sense (I) and reversed primer (II) strands and suitable templates that are designed to be used by DNA polymerases as primers for enzymatic amplification (Fig. 5). ${ }^{33}$ In detail, the approach was based on the combination of a covalent branch point 1 that was accessible via standard ODN synthesis. ${ }^{34}$ With this synthetic strategy it was possible to generate branched

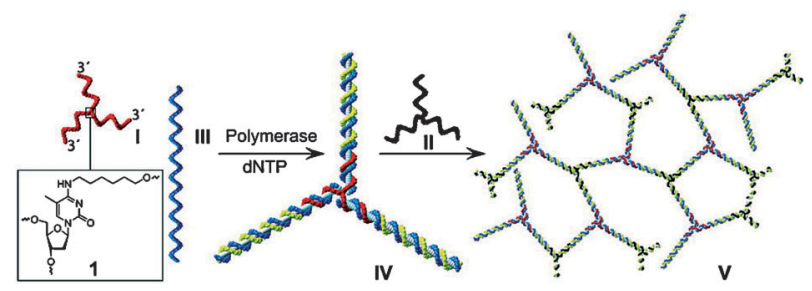

Fig. 5 Schematic depiction of the generation of DNA networks by PCR amplification using branched primer strands ${ }^{33}$ (reproduced with permission from ref. 33). primers I and II bearing three DNA strands that terminate with free $3^{\prime}-\mathrm{OH}$ groups ready for extension by a DNA polymerase. Here, Taq DNA polymerase demonstrated the potential to elongate the primer to an enlarged Y-motif IV and amplify the used template III resulting in DNA networks $\mathbf{V}$ (Fig. 5). The approach is flexible, allowing tuning of the meshes of the network by variation of the template size which was observed in a related study as well. ${ }^{35}$ Additionally, further diversification can be introduced by the employment of chemically modified nucleotides in PCR allowing the introduction of functionalities and reporter moieties. The generated DNA networks were biodegradable as illustrated by restriction digestion. ${ }^{33}$ This result could not be predicted since the sterical hindrance by the multiple branched networks may influence the restriction enzyme and hamper effective digestion. The reported method bears the potential for generating DNA-based networks up to macroscopic dimensions with the action of enzyme catalysed growth and amplification. Further progress in that field could allow access to a DNA-based material useful for tissue engineering.

\section{Enzymatic replication of nanostructures}

Many DNA-based nanostructures, especially geometric DNA objects, are generated out of ssDNA hybridised in a welldefined manner into highly ordered structures. As mentioned above, main progress in this field is based on the successful use of branched DNA junctions as building blocks for nanoconstruction. ${ }^{36}$ The access to large nanostructures in this field is limited by the amount of DNA oligomers and the scale of generated nanostructures. A straightforward approach to solve this problem would be the enzymatic replication and amplification of the DNA nanojunctions. However, the replication of nanostructures is rather difficult since DNA polymerases have to pass stable secondary structures. One approach reported by Joyce and co-workers showed a possible way for the design and synthesis of geometric DNA nanostructures with a limited number of small ODNs. ${ }^{37}$ They used small ODNs acting as helper strands for folding a $1.7 \mathrm{~kb}$ DNA strand into an octahedron (Fig. 6A). Challenging was the synthesis and amplification of the scaffolding long DNA strand (Fig. 6B). For a thermo-stable folding of a long DNA strand with short ODN, the scaffolding long DNA strand has to be single stranded. Therefore, they prepared a $1.7 \mathrm{~kb}$ template using small ODNs in a PCR-based assembly to end up with a ligase mediated dsDNA construct bearing the necessary sequences for correct folding of the stable secondary structures paranemic-crossover ${ }^{38}$ (PX) and double-crossover ${ }^{39}$ (DX) (Fig. 6B).

Rolling circle amplification (RCA) was found to be a potent method for the amplification of nanojunctions as demonstrated in a work by Yan and co-workers (Fig. 7). ${ }^{40}$ RCA is isothermal and, therefore, does not influence the nanojunction folding. The use of DNA polymerases is demanding because they may not pass stable secondary structures. However, Ф29 DNA polymerase was found to be potent for this approach. ${ }^{41}$ This enzyme is known to overcome certain topological constraints such as circular padlock DNA probes hybridised with a linear DNA target or connected to a circular DNA 


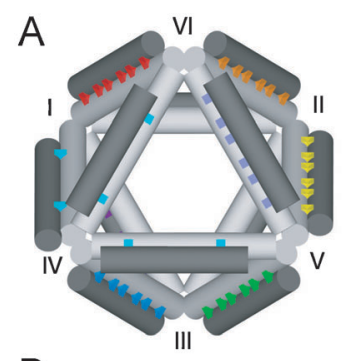

B
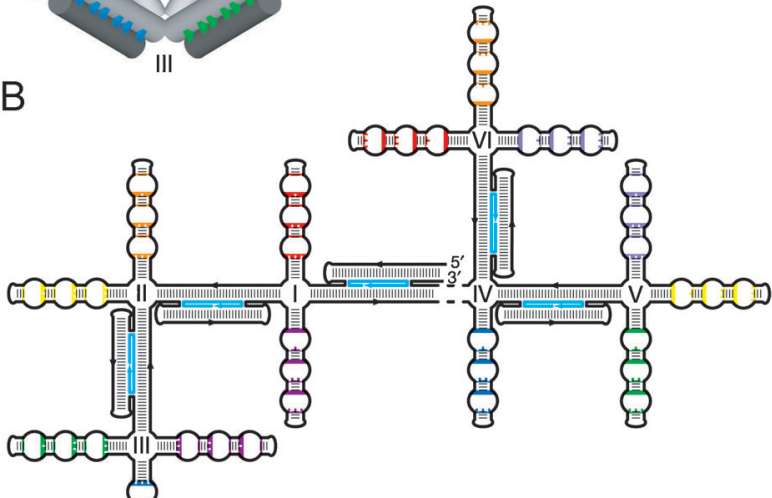

是

Fig. 6 Design of the DNA octahedron. (A) Three-dimensional structure involving twelve ODNs (octahedron edges) connected by six helper strands (octahedron vertices). Five of the ODNs are DX motifs (cyan) and seven are PX motifs (rainbow colours). The joints are four-way junctions that connect the core-layer double helices of each ODN. (B) Secondary structure of the branched-tree folding intermediate. The structure consists of a single heavy chain (black) and five unique light chains (cyan). Like colours indicate half-PX loops whose sequence-specific cross-association generates a strut that serves as an edge of the DNA octahedron. Coloured stripes coincide with strand crossover positions. Folding to the structure in the upper left is complete when all seven PX motifs have formed ${ }^{37}$ (reprinted by permission from ref. 37).

strand. In the depicted study a four-arm nanojunction was chosen as a target to be replicated (Fig. 7). This nanojunction was amplified successfully in multiple repeats. The structure was subsequently liberated by a restriction enzyme PstI. Thereby, many copies of the nanojunction were released. Since the complementary sequence (antisense (-) strand) of the nanojunction was generated, a second round of RCA was needed to finally get the original sequence context resulting in a sense $(+)$ strand.

These findings showed that it is possible to amplify a simple nanojunction with $\Phi 29$ DNA polymerase. In a following report by Lin et al. the amplification of a more complex structure was shown adapting the concept depicted in Fig. 7. ${ }^{42}$ As a substrate for the amplification the known PX-motif, a DNA structure built out of several DNA strands that are crossed over for rigidifying the object, was chosen because it is frequently used in structural DNA nanotechnology. Compared to the structure depicted in Fig. 7, the structural properties of the PX-motif are more challenging for replication by $\Phi 29$ DNA polymerase. The hybridisation density of the PX-motif results in a compact and circular template for RCA. An efficient replication was strongly dependent on the used primer length. For example, using a 60 mer primer the PX-motif was

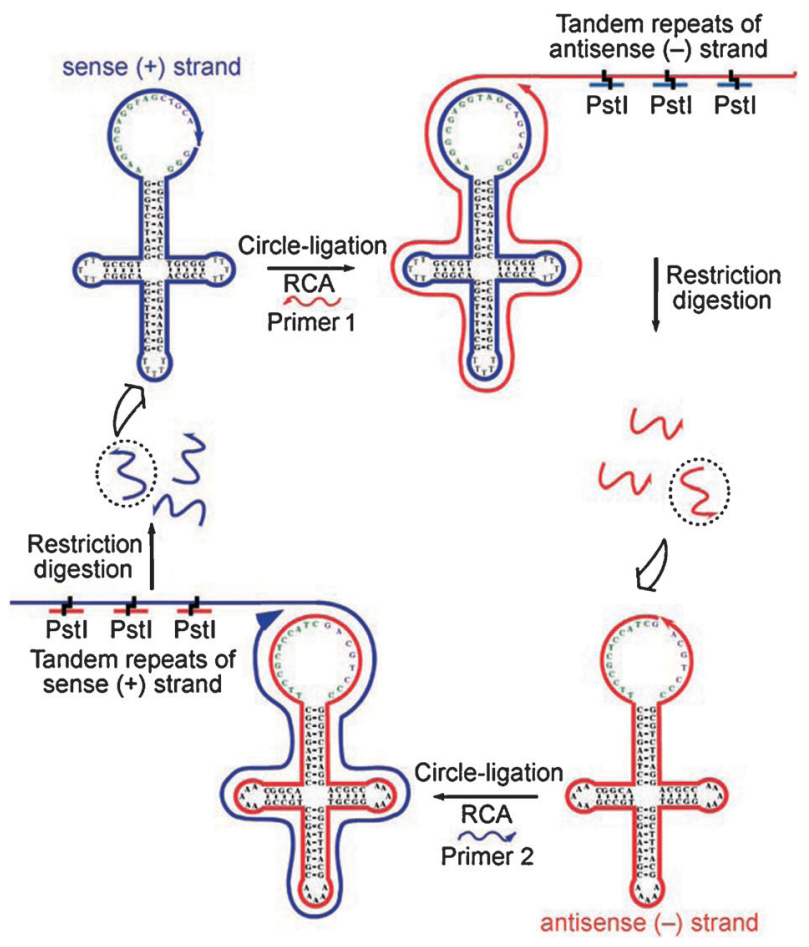

Fig. 7 Schematic diagram of the DNA nanojunction amplification cycle by $\mathrm{RCA}^{40}$ (reproduced with permission from ref. 40).

replicated efficiently with sequence fidelity. Thus, the approach to use RCA to amplify nanostructures demonstrated its potential. Problems due to complex topologies of the nanostructures are still restricting the replication efficiency and limit the application of this approach.

The success in the enzymatic replication of DNA nanostructures brought up the question if replication was also possible in cells or viruses. A concept was presented by Lin and co-workers illustrating the possibility of in vivo amplification of DNA nanostructures. ${ }^{43}$ Related to a former work of Lin mentioned above, a four-armed nanojunction and a PX-motif were chosen and inserted in phagemids, transformed into XL1-Blue cells and amplified in vivo in the presence of helper phages (Fig. 8). This approach uses standard molecular biological techniques leading to a high copy number of the cloned nanostructures. In detail, an ss PX-DNA was inserted into a ds phagemid vector bearing an ss M13 DNA replication origin. The vector was transferred in E. coli cells (XL1-Blue). A single colony of the $E$. coli cells is then cultured before the phagecontaining $E$. coli gets infected by a helper phage. The infected cells were then amplified and the phages were precipitated (Fig. 8). Phage DNA was extracted and restricted. The exponential growth of the phage resulted in a high number of copies of the DNA nanostructure. Comparing with the beforementioned in vitro amplification method, in vivo replication provides a higher efficiency by taking advantage of the naturally existing cellular machinery. A further advantage of in vivo replication is the little amount needed to start the replication process, and once correctly inserted in a vector, it can be easily stored and is ready to be amplified. However, in vivo replication was only performed for rather simple secondary structures. 


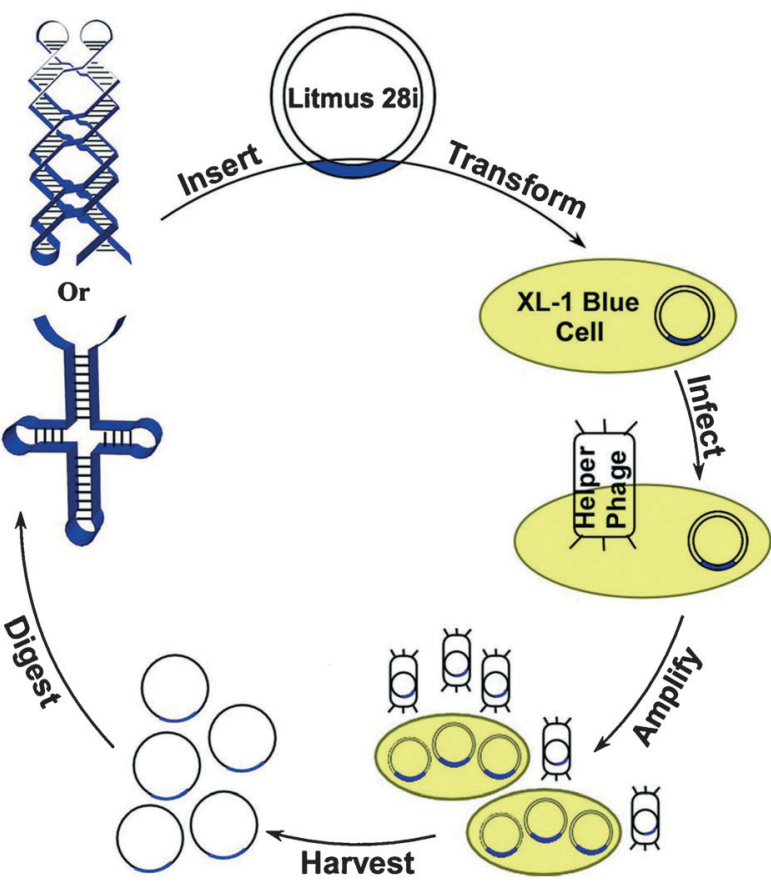

Fig. 8 Schematic drawing showing the in vivo replication of a DNA nanostructure. A single-stranded DNA nanostructure (four-arm nanojunction or PX-motif) is inserted into phagemid, transformed into XL1-Blue cells, and amplified in vivo in the presence of helper phages. High copy numbers of cloned nanostructures can be obtained readily by using standard molecular biology techniques ${ }^{43}$ (reprinted by permission from ref. 43).

Complex or even knotted topologies seem to be a great challenge for the replication machinery in cells. Nevertheless, the success of this method and the straightforward molecular biological methods bear the potential to be commonly used for preparing larger amounts of long ssDNA strands with designed sequences. Further, this approach provides the basis for studies concerning the existence of unknown branched DNA structures in living systems.

\section{Conclusions}

Nowadays, architectural building blocks out of DNA can routinely be designed for construction of objects and assemblies. In this review we aim at the illustration of the impact of enzymes on the construction and amplification of DNA based objects and assemblies. Despite the inherent potential of enzymatic manipulations this issue is only at the beginning of being fully explored. However, the toolbox of enzymes already demonstrated their potential for selective, mild and efficient manipulations. Known enzymatic reactions were successfully transferred to reaction on surface-immobilised DNA and DNA modified Au-NP. DNA assembly controlled by specific enzymatic manipulations will become a useful platform for further applications in the field of programmed structures of biodegradable DNA-based materials. Upscaling processes were supposed to be handled by biotechnological production of enzymes and DNA. Furthermore, the enzymes can be adapted towards their suitability in nanotechnology by protein engineering such as site directed mutation or directed evolution. ${ }^{44}$

\section{References}

1 N. C. Seeman, J. Biomol. Struct. Dyn., 1990, 8, 573-581.

2 N. C. Seeman, Annu. Rev. Biochem., 2010, 79, 65-87.

3 U. Feldkamp and C. M. Niemeyer, Angew. Chem., Int. Ed., 2006, 45, 1856-1876.

4 B. Ding, R. Sha and N. C. Seeman, J. Am. Chem. Soc., 2004, 126, 10230-10231.

5 F. A. Aldaye, P. K. Lo, P. Karam, C. K. McLaughlin, G. Cosa and H. F. Sleiman, Nat. Nanotechnol., 2009, 4, 349-352.

6 T. Li and K. C. Nicolaou, Nature, 1994, 369, 218-221.

7 J. Hyun, J. Kim, S. L. Craig and A. Chilkoti, J. Am. Chem. Soc., 2004, 126, 4770-4771.

8 D. C. Chow, W.-K. Lee, S. Zauscher and A. Chilkoti, J. Am. Chem. Soc., 2005, 127, 14122-14123.

9 B. Basnar and I. Willner, Small, 2009, 5, 28-44.

10 D. C. Chow and A. Chilkoti, Langmuir, 2007, 23, 11712-11717.

11 M. Castronovo, S. Radovic, C. Grunwald, L. Casalis, M. Morgante and G. Scoles, Nano Lett., 2008, 8, 4140-4145.

12 R. Kaufmann, D. Peled, R. Naaman and S. S. Daube, ACS Appl. Mater. Interfaces, 2009, 1, 2320-2324.

13 C. A. Mirkin, R. L. Letsinger, R. C. Mucic and J. J. Storhoff, Nature, 1996, 382, 607-609.

14 A. G. Kanaras, Z. Wang, A. D. Bates, R. Cosstick and M. Brust, Angew. Chem., Int. Ed., 2003, 42, 191-194.

15 A. Kanaras, Z. Wang, M. Brust, R. Cosstick and A. Bates, Small, 2007, 3, 590-594.

16 I. I. S. Lim, U. Chandrachud, L. Wang, S. Gal and C.-J. Zhong, Anal. Chem., 2008, 80, 6038-6044.

17 S. R. Nicewarner Peña, S. Raina, G. P. Goodrich, N. V. Fedoroff and C. D. Keating, J. Am. Chem. Soc., 2002, 124, $7314-7323$.

18 S. A. Claridge, A. J. Mastroianni, Y. B. Au, H. W. Liang, C. M. Micheel, J. M. J. Fréchet and A. P. Alivisatos, J. Am. Chem. Soc., 2008, 130, 9598-9605.

19 W. Zhao, Y. Gao, S. A. Kandadai, M. A. Brook and Y. Li, Angew. Chem., Int. Ed., 2006, 45, 2409-2413.

20 O. I. Wilner, S. Shimron, Y. Weizmann, Z.-G. Wang and I. Willner, Nano Lett., 2009, 9, 2040-2043.

21 M. Kwak and A. Herrmann, Angew. Chem., Int. Ed., 2010, 49, $8574-8587$.

22 K. Ding, F. E. Alemdaroglu, M. Börsch, R. Berger and A. Herrmann, Angew. Chem., Int. Ed., 2007, 46, 1172-1175.

23 J. H. Jeong and T. G. Park, Bioconjugate Chem., 2001, 12, 917-923.

24 F. E. Alemdaroglu, N. Alemdaroglu, P. Langguth and A. Herrmann, Adv. Mater., 2008, 20, 899-902.

25 M. Kwak, I. J. Minten, D.-M. Anaya, A. J. Musser, M. Brasch, R. J. M. Nolte, K. Müllen, J. J. L. M. Cornelissen and A. Herrmann, J. Am. Chem. Soc., 2010, 132, 7834-7835.

26 F. E. Alemdaroglu, J. Wang, M. Börsch, R. Berger and A. Herrmann, Angew. Chem., Int. Ed., 2008, 47, 974-976.

27 J. Wang, F. E. Alemdaroglu, D. K. Prusty, A. Herrmann and R. Berger, Macromolecules, 2008, 41, 2914-2919.

28 N. C. Seeman, J. Theor. Biol., 1982, 99, 237-247.

29 C. Lin, Y. Liu, S. Rinker and H. Yan, ChemPhysChem, 2006, 7, 1641-1647.

30 Y. Li, Y. D. Tseng, S. Y. Kwon, L. d'Espaux, J. S. Bunch, P. L. McEuen and D. Luo, Nat. Mater., 2004, 3, 38-42.

31 S. H. Um, J. B. Lee, N. Park, S. Y. Kwon, C. C. Umbach and D. Luo, Nat. Mater., 2006, 5, 797-801.

32 N. Park, S. H. Um, H. Funabashi, J. Xu and D. Luo, Nat. Mater., $2009,8,432-437$

33 S. Keller, J. Wang, M. Chandra, R. Berger and A. Marx, J. Am. Chem. Soc., 2008, 130, 13188-13189.

34 M. Chandra, S. Keller, C. Gloeckner, B. Bornemann and A. Marx, Chem.-Eur. J., 2007, 13, 3558-3564.

35 Y. H. Jung, Y. S. Chi, M. R. Kim, H. M. Lee, I. S. Choi and Y. G. Kim, Bull. Korean Chem. Soc., 2009, 30 $1365-1367$.

36 N. C. Seeman, Nature, 2003, 421, 427-431.

37 W. M. Shih, J. D. Quispe and G. F. Joyce, Nature, 2004, 427, 618-621.

38 X. Zhang, H. Yan, Z. Shen and N. C. Seeman, J. Am. Chem. Soc., 2002, 124, 12940-12941. 
39 X. J. Li, X. P. Yang, J. Qi and N. C. Seeman, J. Am. Chem. Soc., 1996, 118, 6131-6140.

40 C. Lin, M. Xie, J. J. L. Chen, Y. Liu and H. Yan, Angew. Chem., Int. Ed., 2006, 45, 7537-7539.

41 W. Zhao, M. M. Ali, M. A. Brook and Y. Li, Angew. Chem., Int. Ed., 2008, 47, 6330-6337.
42 C. Lin, X. Wang, Y. Liu, N. C. Seeman and H. Yan, J. Am. Chem. Soc., 2007, 129, 14475-14481.

43 C. Lin, S. Rinker, X. Wang, Y. Liu, N. C. Seeman and H. Yan, Proc. Natl. Acad. Sci. U. S. A., 2008, 105, 17626-17631.

44 S. Brakmann and K. P. Johnsson, Directed Molecular Evolution of Proteins, Wiley-VCH Verlag GmbH, Weinheim, 2002. 